\title{
Ground-State Multiplicities and $d-d$ Excitations of Transition-Metal Complexes by Effective Hamiltonian Method
}

\section{A. V. SOUDACKOV*}

Fachbereich Theoretische Chemie, Universität Hannover, Am Kleinen Felde 30, 30167, Hannover, Germany, and Karpov Institute of Physical Chemistry, Vorontsovo pole, 10, 103064,

Moscow, Russia

\section{A. L. TCHOUGRÉEFF}

Max-Planck-Institut für Physik komplexer Systeme, Bayreuther Str. 40-16, 01187, Dresden, Germany, and Karpov Institute of Physical Chemistry, Vorontsovo pole, 10, 103064, Moscow, Russia

\section{A. MISURKIN}

Karpov Institute of Physical Chemistry, Vorontsovo pole, 10, 103064, Moscow, Russia

Received November 28, 1994; revised manuscript received April 6, 1995; accepted April 24, 1995

\begin{abstract}
Magnetic and optical properties of transition-metal complexes are governed by the ground state and the low-energy excitation spectrum of the $d$-shell of the central transition metal ion. These spectra are successfully fit to the crystal field theory. We present here an account of the effective Hamiltonian method recently developed to calculate the ground state and the excitations of the $d$-shells of transition-metal complexes and report the results of its application to some complexes of particular interest. (c) 1996 John Wiley \& Sons, Inc.
\end{abstract}

\footnotetext{
*Alexander von Humboldt Fellow.
} 


\section{Introduction}

$\mathbf{T}$ ransition-metal complexes (TMC) are "large systems" for direct quantum chemical treatment. Nevertheless, both semiempirical [1-5] and $\mathrm{ab}$ initio [6-13] methods based upon the Hartree-Fock-Roothaan approximation have been used in order to calculate the ground states and the excitation spectrum of TMC. With respect to the ground state of $\mathrm{TMC}$, the problems of correct reproduction of its spin multiplicity and spatial symmetry are particularly difficult (see below). In the case of $\mathrm{ab}$ initio calculations with large $\mathrm{Cl}$ expansions [6-10] or with a special selection of configurations in the MCSCF wave function [11, 12], a reasonable agreement of the calculated ground-state spin and symmetry and of transition energies with experiment can be obtained. However, such calculations are restricted to relatively simple systems.

Recently, a semiempirical INDO/s-CI method for the calculation of the electronic transition energies was elaborated and extended to transition-metal atoms [2]. It successfully applies to rather complex systems like ferrocene [3] or porphyrin complexes of transition metals $[4,5]$. However, despite reasonable numerical agreement between the calculated and observed $d-d$ transition energies obtained by this method, some fundamental problems remain unsolved. The success in [2-5] was achieved by a heavy loss of the broken Aufbauprinzip and (as a consequence of the latter) by the loss of the confidence that the obtained Slater determinants represent the minima of the Hartree-Fock energy functional rather than saddle points.

The main complication which arises when semiempirical quantum chemistry addresses TMC is the doubt in the validity of the fundamental quantum chemical approximation, the self-consistent-field (SCF) approximation, for this class of the molecules. The main problems the SCF approximation encounters are the following (for more detailed discussion, see [14] and references therein):

(i) The Koopman's theorem is not valid for the states with large contributions from the atomic $d$-states;

(ii) the Aufbauprinzip frequently breaks for the orbitals with significant contributions from the atomic $d$-states; and

(iii) the iteration procedures implied by the SCF approximation converge very slowly or oscillate.

The breaking of the Koopman's theorem is the most spectacular among the problems listed above. This theorem appears as a corollary of the SCF approximation where each electron is treated as if it moved in a mean field induced by nuclei and other electrons. The ionization potentials are then equal to minus energies of electrons in such a potential (minus orbital energies). This picture is an approximation since the mean field itself changes when an electron is removed from the system or added to it. The difference between the minus orbital energy which must be the ionization potential according to the Koopmans' theorem and the real ionization potential is called the relaxation energy. For organic molecules, the relaxation energies are usually small and the whole picture remains consistent. In the case of $\mathrm{TMC}$, the relaxation energies can reach the values from 10 to $20 \mathrm{eV}$ when the levels with some significant contribution from $d$-orbitals are involved [15]. This suggests that the real behavior of $d$-electrons in TMC does not fit in the picture where independent electrons move in the mean field induced by the nuclei and other electrons. By contrast, electrons trace any motion of each other very carefully. In other words, $d$-electrons are strongly correlated.

Another problem, which is rarely recognized as such, is the break of the Aufbauprinzip (the rule of the occupation of MOs by electrons by two from the bottom), which frequently happens in the semiempirical calculations on TMC. In this case, as in the case of the Koopmans' theorem, the orbitals which turn out to be unoccupied or singly occupied but have lower orbital energy than some doubly occupied orbitals are the orbitals formed largely by the $d$ atomic orbitals. One can check (see [16] and the Appendix) that the Slater determinants breaking the Aufbauprinzip do not present a minimum of the Hartree-Fock energy functional but a saddle point. This in its turn causes the well-known but rarely reported problems with the convergency of the SCF iterations. However, more important than the convergency problems is the doubtfulness of the very idea to parametrize a semiempirical Fock operator for transition metals using, throughout, the calculation a trial wave function which is not a stable solution of the SCF problem. 
From the above discussion, we see that there are some fundamental problems in the semiempirical description of the electronic structure of TMC. They appear due to the relatively important role of the correlations which manifest themselves primarily in the $d$-shells, whereas the description of electrons in the ligands seems to be less problematic. At the same time, $d$-electrons are responsible for the ground-state total spin and for the lowenergy excitations of TMC.

The instability of the Hartree-Fock solutions for TMC does not create any physical problem by itself. It simply indicates that the ground state cannot be presented by a single Slater determinant and some other configurations must be added. The problem which arises in this concern is that the $\mathrm{CI}$ series using the Hartree-Fock orbitals as a one-electron basis set converge rather slowly and thus a large number of configurations must be taken. A truly large $\mathrm{CI}$ expansion once obtained is very difficult to interpret since none of the configurations dominates it. For that reason, any qualitative picture of the electronic structure of TMC gets lost.

This gloomy picture is, however, in a sharp contrast with a real state of things with the physical understanding of the electronic structure of TMC, which is, indeed, fairly transparent. The experimental data concerning the $d-d$ excitation spectra of TMC can be successfully fit to the models equivalent to the crystal field theory (CFT) [17]. That means that specific properties of TMC are determined by the $d$-electrons of the transitionmetal ion. Their low-energy excitations are responsible for the characteristic absorption bands in the optical spectra and for the magnetic properties. The ground-state spin depends on the balance between the electron repulsion of $d$-electrons and their interaction with the ligands which provide some external field. The excitations of the ligands have much larger energies than those in the $d$-shell and, incidentally, the ligands have closed electronic shells so that they do not contribute directly to the spin multiplicity of the complex. The features formulated above apparently correspond to the situation covered by the naive CFT where all the interesting events happen in the $d$-shell of transition-metal ions whose ligand environment remains inert [17]. The qualitative physical picture provided by the CFT is correct to a large extent. The major part of magnetic and optical experiments on $\mathrm{TMC}$, indeed, can be interpreted in the framework of the CFT operating with the multiplets of some fixed number of $d$-electrons in the external field of appropriate symmetry (see, e.g., [18]). The CFT is that successful due to the correct form of the electronic wave function that it implies. Obviously, the wave function of a complex in CFT is a product of the multiplet (full $\mathrm{cI}$ ) state for some fixed number of $d$-electrons and of an unspecified closed-shell state of the remaining electrons in the ligands. This remainder was never considered explicitly (see, however, below) and that is why the crystal fields could not be satisfactorily calculated within the CFT's own framework.

In the effective Hamiltonian method [14], the two most important features of the electronic structure of TMC outlined above, namely, the presence of an isolated group of strongly correlated $d$-electrons on the metal atom and the existence of the closed-shell ligands, were taken into account explicitly. The distinction between the ligands and the $d$-shell of TMC is used in order to describe $d$-electrons and electrons in the ligand orbitals, employing different levels of accounting for the electron correlations. The following features were implemented in the method: (1) the multiplet structure of $d$-electrons in the central ion (to do so, the configuration interaction $[\mathrm{Cl}]$ is completely taken into account in the $d$-shell); (2) electronic structure of the ligands is treated realistically, but the single-determinant approximation suffices for that; and (3) the weak covalency resulting from the metal-ligand interactions is included by the effective Hamiltonian technique.

The method has been parametrized and then applied to the metal fluorine complexes, metal hexahydrate and hexamine complexes, metal hexachloro and tetrachloro complexes, metallocenes, and mixed ligand complexes of the $D_{4 h}$ symmetry [19-22]. In all the cases studied, we observed perfect agreement between the experimental data concerning the spin and symmetry of the ground state of the complex and our calculations. The spectra of the $d-d$ excitations were reproduced within the accuracy of $2000 \mathrm{~cm}^{-1}$. In the present article, we apply the effective Hamiltonian-crystal field (EHCF) method [14] which is briefly described for some complexes with nitrogen-containing ligands including porphin which are "truly large systems."

\section{Account of the Method}

The formal derivation of the EHCF method proceeds as follows [14]: We separate the whole set of 
the valence atomic orbitals (AOs) of the TMC (it includes the $4 s^{-}, 4 p-$, and $3 d$-AOs of the metal and the valence $A O S$ of the ligand atoms) into two parts. The first part contains only $3 d$-orbitals of the transition-metal atom ( $d$-subsystem). The second part contains $4 s^{-}$and $4 p$-AOs of the transition metal and the valence orbitals of the ligand atoms (ligand subsystem or $l$-subsystem). Then, the total Hamiltonian for a TMC can be represented as a sum:

$$
H=H_{d}+H_{l}+H_{c}+H_{r},
$$

where $H_{d}$ is the Hamiltonian for the $d$-electrons in the field of the atomic cores of TMC; $H_{l}$, the Hamiltonian for the electrons of the $l$-subsystem; and $H_{c}$ and $H_{r}$, respectively, the Coulomb and the resonance interaction operators between the two subsystems.

For the most of TMC with closed-shell ligands, the excitations in the ligands are very high in energy as compared to the $d-d$ excitations, and, thus, their contribution is negligible. The ground state of the $l$-subsystem can be described by a Slater determinant $\Phi_{l}\left({ }^{1} A_{1}\right)$ with zero total spin. The wave function $\Phi_{n}$ for the $n$-th electronic state of a TMC then takes the form

$$
\Phi_{n}\left(\sum_{k} C_{k}^{n}\left|n_{d} k\right\rangle\right) \wedge \Phi_{l}=\Phi_{d}^{n} \wedge \Phi_{l},
$$

where $\left|n_{d} k\right\rangle$ are the spin- and symmetry-adapted $n_{d}$-electron wave functions constructed on the metal $d$-orbitals, and $C_{k}^{n}$ are variation parameters. Both the spin multiplicity and the point symmetry of the functions of this type coincide with the multiplicity and the symmetry of the functions $\Phi_{d}^{n}$ of the $d$-subsystem.

The wave functions of the type Eq. (2) correspond to a fixed integer number $\left(n_{d}\right)$ of electrons in the $d$-shell of the metal ion. It is not that bad from the point of view of the description of electron distribution, since for most of the complexes, the total charge transfer between the $d$-shell and the ligands usually does not exceed a few hundredths of the unit charge. A more serious problem is that all the matrix elements of the resonance (electron-hopping) operator $H_{r}$ calculated with the functions of that type are vanishing. That prevents any correct description of the interaction between the ligands and the $d$-shell within the above class of the trial wave functions if the Hamiltonian Eq. (1) is used. To include the effects of the resonance interaction between the subsystems, we consider the effective Hamiltonian $H^{e f f}$ [14] which operates in the subspace spanned by the functions Eq. (2). Its eigenvalues coincide with those of the exact Hamiltonian Eq. (1):

$$
\begin{aligned}
H^{e f f} & =P H_{0} P+H_{R R} \\
H_{0} & =H_{d}+H_{l}+H_{c} \\
H_{R R} & =P H_{r} Q\left(E Q-Q H_{0} Q\right)^{-1} Q H_{r} P .
\end{aligned}
$$

Here, $P$ is the projection operator on the subspace of functions with fixed number of $d$-electrons; $Q$ $=1-P$.

The variational problem for the effective Hamiltonian $H^{e f f}$ Eq. (3) within the subspace spanned by the wave functions of the type Eq. (2) splits into a pair of interconnected equations for the functions $\Phi_{d}^{n}$ and $\Phi_{l}$ (see, e.g., $[14,23]$ ):

$$
\begin{aligned}
H_{d}^{e f f} \Phi_{d}^{n} & =E_{d}^{n} \Phi_{d}^{n} \\
H_{l}^{e f f} \phi_{l} & =E_{l} \Phi_{l},
\end{aligned}
$$

with the effective Hamiltonians for the subsystems given by

$$
\begin{aligned}
& H_{d}^{e f f}=H_{d}+\left\langle\Phi_{l}\left|H_{c}+H_{R R}\right| \Phi_{l}\right\rangle \\
& H_{l}^{e f f}=H_{l}+\left\langle\Phi_{d}^{n}\left|H_{c}+H_{R R}\right| \Phi_{\mathfrak{d}}^{n}\right\rangle .
\end{aligned}
$$

Since the l-subsystem is described by a single Slater determinant $\Phi_{l}$, the latter must be found from the self-consistent-field procedure applied to the Fockian $F_{l}^{e f f}$ derived from the Hamiltonian $H_{l}^{\text {eff }}$ by the standard method [23, 24]. Proceeding semiempirically, we apply the standard CNDO parametrization [24] to all ligand atoms. The core attraction parameters $U_{s s}$ and $U_{p p}$ of the metal $4 s^{-}$ and $4 p$-orbitals and the metal core charge $Z_{M}$ are renormalized according to

$$
\begin{aligned}
U_{s s} & \rightarrow U_{s s}+n_{d} g_{s d} \\
U_{p p} & \rightarrow U_{p p}+n_{d} \bar{g}_{p d} \\
Z_{M} & \rightarrow Z_{M}-n_{d} .
\end{aligned}
$$

Here, $g_{s d}=(s s \mid d d)-(s d \mid d s) / 2 ; \bar{g}_{p d}$ is the average of the integrals $g_{i \mu}$, where $i=4 p_{x}, 4 p_{y}, 4 p_{z}$ and $\mu=3 d_{z^{2}}, 3 d_{x z}, 3 d_{y z}, 3 d_{x^{2}-y^{2}}, 3 d_{x y}$. One can check $[14,16]$ that the renormalization according to Eq. (6) finally allows one to use the standard values of the orbital electronegativities for the $4 s$ and $4 p$-AOs given in [25]. 
The solution of the Hartree-Fock problem for the $l$-subsystem with the above approximate CNDO Fockian gives the one-electron density matrix $P_{k l}$, the energies of the molecular orbitals (MOS) $\varepsilon_{i}$, and MO LCAO coefficients $c_{i k}$ [24]. These quantities completely describe the electronic structure of the $l$ subsystem within the accepted approximation. They are used to construct the effective Hamiltonian $H_{d}^{\text {eff }}$ Eq. (5) in the following way: The operators $H_{c}$ and $H_{R R}$ are averaged over the ground state $\Phi_{l}$ of the $l$-subsystem which yields the effective Hamiltonian $H_{d}^{\text {eff }}$ of the form [14]

$$
\begin{aligned}
H_{d}^{e f f}= & \sum_{\mu \nu \sigma} U_{\mu \nu}^{e f f} d_{\mu \sigma}^{+} d_{\nu \sigma} \\
& +\frac{1}{2} \sum_{\mu \nu \rho \eta} \sum_{\sigma \tau}(\mu \nu \mid \rho \eta) d_{\mu \sigma}^{+} d_{\nu \sigma} d_{\rho \tau}^{+} d_{\eta \tau},
\end{aligned}
$$

where the Coulomb interaction between $d$ electrons is taken as in the free ion and the effective core attraction parameters for metal $d$-electrons $U_{\mu \nu}^{e f f}$ contain the corrections originating both from the Coulomb and the resonance interaction of $d$-electrons with the $l$-subsystem:

$$
U_{\mu \nu}^{e f f}=\delta_{\mu \nu} U_{d d}+W_{\mu \nu}^{\text {atom }}+W_{\mu \nu}^{\text {field }}+W_{\mu \nu}^{\text {cov }},
$$

where

$$
W_{\mu \nu}^{\text {atom }}=\delta_{\mu \nu}\left(\sum_{i \in S, p} g_{\mu i} P_{i i}\right)
$$

is the repulsion of electrons in the $d$-shell from those in the $4 s-$ and $4 p$-AOs of the central metal ion;

$$
W_{\mu \nu}^{\text {field }}=\sum_{L}\left(P_{L L}-Z_{L}\right) V_{\mu \nu}^{L}
$$

is the Coulomb interaction of the $d$-electrons with the net charges on the ligand atoms, having the standard CFT form, and

$$
W_{\mu \nu}^{\text {cov }}=-\sum_{i} \beta_{\mu i} \beta_{\nu i}\left(\frac{\left(1-n_{i} / 2\right)}{\Delta E_{d i}}-\frac{\left(n_{i} / 2\right)}{\Delta E_{i d}}\right)
$$

comes from the resonance. Here, $P_{i i}$ is the diagonal matrix element of the one-electron density matrix of the ligand subsystem; $P_{L L}=\sum_{l \in L} P_{l l}$, the electronic population on the ligand atom $L_{;} Z_{L}$, the core charge of the ligand atom $L_{i} V_{\mu \nu}^{L}$, the matrix element of the potential energy operator describing the interaction between a $d$-electron and an electron placed on the ligand atom $L ; n_{i}$, the occupation number of the $i$-th ligand мо $\left(n_{i}=0\right.$ or 2); $\Delta E_{d i}\left(\Delta E_{i d}\right)$, the energy which is necessary to transfer an electron from the $d$-shell (from the $i$-th MO) to the $i$-th MO (to the $d$-shell); and $\beta_{\mu i}$, the resonance integral between the $\mu$-th $d$-orbital and the $i$-th ligand MO which is expressed through the resonance integrals $\beta_{\mu k}$ between the $\mu$-th $d$-orbital and the $k$-th ligand $\mathrm{AO}$ :

$$
\beta_{\mu k}=-\left(I_{d}+I_{k}\right) S_{\mu k} \beta^{M L},
$$

where $I_{d}$ and $I_{k}$ are the valence state ionization potentials; $S_{\mu k}$, the overlap integral between the $\mu$-th $d$-AO and the $k$-th ligand $\mathrm{AO}$; and $\beta^{M L}$, the only adjustable parameter specific for each $M L$ pair, where $M$ stands for a transition metal atom and $L$ stands for a donor atom in the ligand (like nitrogen, oxygen, etc.).

The effective Hamiltonian for the $d$-shell Eq. (7) obviously has the form of the standard crystal field Hamiltonian. The covalence term dominating the $d$-level splitting is apparently analogous by its origin to the ligand-field parameters of the angular overlap model (АOM) [26]. The important difference between them is that in the AOM all the ligand-field parameters are fit to the experimental spectra for each ligand and are not transferable from one ligand to another even if the donor atom is the same. In our approach, the electronic structure of the ligands is taken into account explicitly. That allowed us to parametrize (see [14, 19-22] and below) only the magnitude of the hopping between the orbitals of the given donor atom and $d$-orbitals of the given transition metal. The same value can be used for all the ligands containing a given donor atom, thus permitting one to reproduce the effects of the substitution in the ligands on the crystal field induced on the $d$-shell.

After the effective Hamiltonian for $d$-electrons is constructed, the states of $n_{d}$ electrons in the $d$-shell are calculated by diagonalizing the matrix associated with $H_{d}^{\text {eff }}$ in the $n_{d}$ electron wavefunction basis set. The ground state of the whole complex is then obtained by taking the external product of the ligand Slater determinant and that $n_{d}$-electron wave function obtained by the diagonalization of $H_{d}^{e f f}$ which has the lowest eigenvalue. The $d-d$ excited states (they are the lowenergy excitations of the whole complex) are then obtained by multiplying other $n_{d}$-electron func- 
tions by the same Slater determinant. The corresponding excitation energies are then estimated as the differences between the corresponding eigenvalues of the effective Hamiltonian $H_{d}^{e f f}$.

\section{Calculation Results and Discussion}

In this article, we applied the method described in the previous section to calculate the electronic structure of the ligand sphere and $d$-shells of a series of the $\mathrm{ML}_{4} Z_{2}$ compounds where $\mathrm{M}=\mathrm{Fe}$, $\mathrm{Ni} ; \mathrm{L}=\mathrm{H}_{2} \mathrm{O}, \mathrm{NH}_{3}, \mathrm{Py} ; \mathrm{Z}=\mathrm{H}_{2} \mathrm{O}, \mathrm{NCS}^{-}, \mathrm{Cl}^{-}$, and to metal porphyrins. The spin multiplicity and the spatial symmetry of the ground states of the considered mixed complexes are correctly reproduced by our method (the parameters $\beta^{M L}$ were found from the calculations $[20,21]$ on the corresponding pure complexes $\left(\mathrm{ML}_{6}^{2+}\right.$ and $\left.\mathrm{ML}_{6}^{4-}\right)$.

The FHCF method supplies us with the results of three types: (1) the standard quantum chemical description of the $l$-subsystem (i.e., electron densities on atoms, orbital energies, and the orbital expansion coefficients); (2) the many-electron ground state and the excitations of the $d$-shell; and (3) the information on the particular MOs and the charge-transfer states which largely contribute to the effective crystal field induced by the $l$-system into the $d$-system. Three these types of data are presented in Tables I-VIII. The forms of the MOs and the orbital energies are condensed in the contributions to the splitting, and for that reason, we do not give these data here.

For all the four considered $\mathrm{ML}_{4} \mathrm{Z}_{2}$ complexes, the ground state is in accord with the experiment. It is the ${ }^{3} B_{1 g}$ state for the Ni(II) compounds $[27,28]$ and ${ }^{5} B_{2 g}$ for the Fe(II) mixed complex $[29,30]$. The charge distribution in all four complexes does not contradict to the intuitive ideas concerning the charge distributions. Note that in all the cases our method gives some noticeable positive charge on the metal ion, thus curing an old [25] problem of either very small positive or even negative charges on the central ions. The transition energies of the $\mathrm{Ni}$ compounds are in fair agreement with the experimental assignments [27, 28].

The absorption spectra of $\left[\mathrm{Fe}(\mathrm{py})_{4}(\mathrm{NCS})_{2}\right]$ deserve some more detailed discussion. In accord with the experiment [29], our calculation reproduces two spin-allows transitions ${ }^{5} B_{2 g} \rightarrow{ }^{5} A_{1 g}$, ${ }^{5} B_{2 g} \rightarrow{ }^{5} B_{1 g}$ originating from the octahedral ${ }^{5} T_{2 g} \rightarrow$
TABLE I

Ligand systems of $\mathrm{Ni}(\mathrm{Py})_{4} \mathrm{Cl}_{2}$ and

$\left[\mathrm{Ni}(\mathrm{Py})_{4}\left(\mathrm{H}_{2} \mathrm{O}\right)_{2}\right]^{2+}$.

\begin{tabular}{lcr}
\hline \multicolumn{3}{c}{$\mathrm{Ni}(\mathrm{Py})_{4} \mathrm{Cl}_{2}$} \\
\hline \multicolumn{3}{c}{ Frontier MOs of the l-system } \\
\hline Symmetry & Composition & Energy (eV) \\
\hline $7 e_{g}$ (HOMO) & $p_{\pi}(\mathrm{Cl})$ & -9.554 \\
$3 b_{2 g}($ LUMO) & $\pi=$ MO(Py) & 2.350 \\
\hline \multicolumn{3}{c}{ Metal AOS populations } \\
\hline $4 s$ & $4 p_{x(y)}$ & $4 p_{z}$ \\
0.500 & 0.285 & 0.356 \\
\hline
\end{tabular}

Effective atomic charges

\begin{tabular}{lccccc}
$\mathrm{Ni}$ & $\mathrm{N}$ & $\mathrm{C}_{\text {meta }}$ & $\mathrm{C}_{\text {ortho }}$ & $\mathrm{C}_{\text {para }}$ & $\mathrm{Cl}$ \\
\hline 0.573 & -0.181 & 0.089 & -0.025 & 0.061 & -0.652 \\
\hline
\end{tabular}

$\left[\mathrm{Ni}(\mathrm{Py})_{4}\left(\mathrm{H}_{2} \mathrm{O}\right)_{2}\right]^{2+}$

\begin{tabular}{llc}
\hline & Frontier MOs $^{\mathrm{a}}$ & \\
\hline Symmetry & \multicolumn{1}{c}{ Composition } & Energy (eV) \\
\hline$b_{3 u}(\mathrm{HOMO})$ & $p_{\pi}(\mathrm{O})+p_{\sigma}(\mathrm{N})$ & -19.820 \\
$b_{3 u}(\mathrm{LUMO})$ & $p_{\pi}(\mathrm{N})$ & -4.903 \\
\hline
\end{tabular}

Metal AOs populations

\begin{tabular}{llll}
\hline $4 s$ & $4 p_{x}$ & $4 p_{y}$ & $4 p_{z}$ \\
0.434 & 0.286 & 0.274 & 0.232 \\
\hline
\end{tabular}

Effective atomic charges

\begin{tabular}{lccccc}
\hline $\mathrm{Ni}$ & $\mathrm{N}$ & $\mathrm{C}_{\text {meta }}$ & $\mathrm{C}_{\text {ortho }}$ & $\mathrm{C}_{\text {para }}$ & $\mathrm{O}$ \\
\hline 0.774 & -0.234 & 0.136 & -0.004 & 0.089 & -0.455 \\
\hline \multicolumn{2}{l}{${ }^{\mathrm{a}}$ The l-system has the effective symmetry } & $D_{2 h}$.
\end{tabular}

${ }^{5} E_{8}$ transition split by the tetragonal field. Their order is, however, inverted as compared to the standard assignment scheme [18] used also in [29]. The point symmetry labels ascribed to the excited states $\left({ }^{5} A_{1 g}\right.$ and $\left.{ }^{5} B_{1 g}\right)$ are assigned without strong experimental support. The reasoning given in [29] is based on the idea that the $d_{x^{2}-y^{2}}$ and $d_{z^{2}}$ orbitals singly occupied in the ground state of 
TABLE II

Atomic charges in some complexes.

\begin{tabular}{|c|c|c|c|c|}
\hline \multicolumn{5}{|c|}{$\left[\mathrm{Ni}\left(\mathrm{NH}_{3}\right)_{4}(\mathrm{NCS})_{2}\right]$} \\
\hline $\mathrm{Ni}$ & $\mathrm{N}\left(\mathrm{NH}_{3}\right)$ & $N($ NCS) & C & S \\
\hline 0.680 & -0.281 & -0.381 & 0.198 & -0.518 \\
\hline \multicolumn{5}{|c|}{$\left[\mathrm{Fe}(\mathrm{py})_{4}(\mathrm{NCS})_{2}\right]$} \\
\hline $\mathrm{Fe}$ & $N(p y)$ & $N(\mathrm{NCS})$ & $\mathrm{C}$ & $S$ \\
\hline 0.830 & -0.20 & -0.43 & 0.20 & -0.53 \\
\hline \multicolumn{5}{|c|}{$\mathrm{FeP}$} \\
\hline $\mathrm{Fe}$ & $\mathrm{N}$ & $\mathrm{C}_{\alpha}$ & $\mathrm{C}_{\beta}$ & $\mathrm{C}_{\text {meso }}$ \\
\hline 0.921 & -0.372 & 0.134 & -0.031 & -0.069 \\
\hline \multicolumn{5}{|c|}{ CoP } \\
\hline Co & $N$ & $\mathrm{C}_{\alpha}$ & $\mathrm{C}_{\beta}$ & $\mathrm{C}_{m e s o}$ \\
\hline 0.831 & -0.354 & 0.136 & -0.032 & -0.067 \\
\hline
\end{tabular}

$\left[\mathrm{Fe}(\mathrm{py})_{4}(\mathrm{NCS})_{2}\right]$ are bonding. In this case, the stronger bonding interaction between the iron(II) ion and the NCS ${ }^{-}$ligands (evident from the structural data [14]) results in a lower energy of the $d_{z^{2}}$ orbital as compared to a weaker bonding $d_{x^{2}-y^{2}}$ orbital.

Meanwhile, in our approach, the $d$-orbitals are excluded from the uniform molecular orbital treatment, and, thus, the terms "bonding" or "antibonding" do not apply to them. The $d$-orbitals do not participate in the formation of chemical bonds and are therefore "nonbonding." The relative position of the strongly localized $d$-orbitals on the energy scale is predominantly determined by the destabilizing interaction with the lone pairs of the donor nitrogen atoms of the Py and $\mathrm{NCS}^{-}$ligands, respectively (see Table V). The interaction with the lone pairs of the NCS groups is stronger due to both the geometry reasons affecting the magnitude of the resonance factors $\beta_{i \mu}$ and the differences in the electronic structure of the two ligands. The ionization potential is smaller in the case of the $\mathrm{NCS}^{-}$lone pair and, thus, the energy of the charge-transfer state appearing in the denominator [Eq. (8)] is also smaller, thus assuring stronger destabilization of the $d_{z^{2}}$ orbital as compared to
TABLE III $d-d$ excitation energies for $\mathrm{Ni}\left(\mathrm{NH}_{3}\right)_{4}(\mathrm{NCS})_{2}$ and $\mathrm{Fe}(\mathrm{py})_{4}(\mathrm{NCS})_{2}$.

\begin{tabular}{|c|c|c|}
\hline Transition & $E^{\text {calc }}\left(\mathrm{cm}^{-1}\right)$ & $E^{\text {obs }}\left(\mathrm{cm}^{-1}\right)$ \\
\hline \multicolumn{3}{|c|}{$\left[\mathrm{Fe}(\mathrm{py})_{4}(\mathrm{NCS})_{2}\right]$, ground state ${ }^{5} B_{2 g}{ }^{a}$} \\
\hline${ }^{5} B_{2 g} \rightarrow{ }^{5} E_{g}$ & 1,450 & - \\
\hline$\rightarrow{ }^{5} B_{1 g}$ & 5,160 & 9,880 \\
\hline$\rightarrow{ }^{5} A_{1 g}$ & 10,980 & 11,460 \\
\hline \multicolumn{3}{|c|}{$\mathrm{Ni}\left(\mathrm{NH}_{3}\right)_{4}(\mathrm{NCS})_{2}$, ground state ${ }^{3} \mathrm{~B}_{1 \mathrm{~g}}{ }^{\mathrm{b}}$} \\
\hline${ }^{3} B_{1 g} \rightarrow{ }^{3} B_{2 g}$ & 8,898 & 10,750 \\
\hline & 9,422 & 10,750 \\
\hline & 13,843 & 13000 \\
\hline$\rightarrow{ }^{1} B_{1 g}$ & $14,372\}$ & 13,000 \\
\hline$\rightarrow{ }^{3} E_{g}$ & 14,913 & 17,350 \\
\hline$\rightarrow{ }^{3} A_{2 g}$ & 15,928 & 17,500 \\
\hline$\rightarrow{ }^{1} B_{2 g}$ & 22,909 & - \\
\hline$\rightarrow^{1} E_{g}$ & 23,290 & - \\
\hline$\rightarrow{ }^{1} A_{1 g}$ & 23,774 & - \\
\hline$\rightarrow{ }^{3} E_{g}$ & 25,482 & 27,900 \\
\hline$\rightarrow{ }^{3} A_{2 g}$ & 26,211 & 28,000 \\
\hline$\rightarrow{ }^{1} E_{g}$ & 26,890 & - \\
\hline$\rightarrow{ }^{1} A_{2 g}$ & 29,687 & - \\
\hline $\begin{array}{l}{ }^{a} \text { The Racah } \\
\text { [22]. } \\
\text { b The Racah } \\
\text { [18]; Experim }\end{array}$ & $\begin{array}{l}\mathrm{s}: B=650 \mathrm{~cm} \\
\mathrm{~s}: B=851 \mathrm{~cm} \\
\text { from [27]. }\end{array}$ & $\begin{array}{l}C=2400 \mathrm{~cm}^{-1} \\
C=4008 \mathrm{~cm}^{-1}\end{array}$ \\
\hline
\end{tabular}

that of the $d_{x^{2}-y^{2}}$ orbital. It should be noted, however, that the calculated splitting between ${ }^{5} A_{18}$ and ${ }^{5} B_{1} g$ states is somewhat overestimated. At the same time, in the case of the NCS complex of Ni, the split between the $d_{x^{2}}$ and $d_{x^{2}-y^{2}}$ orbitals is much less pronounced.

Having in mind the proven success of the EHCF method in predicting the ground-state spin and symmetry for many diverse TMC (including the successful description of the spin-transitions in the Fe(II) complexes with the nitrogen containing ligands reported elsewhere [22]), we undertook an attempt to attack metal porphyrins. The SCF ab initio calculations on metal porphyrins systematically give erroneous results, overestimating the total energy of the high-spin ground states. In the case of MnP ( $\mathrm{P}$ stands hereafter for porphin), the 
TABLE IV

d-d excitations in $\mathrm{Ni}(\mathrm{Py})_{4} \mathrm{Cl}_{2}$ and $\left[\mathrm{Ni}(\mathrm{Py})_{4}\left(\mathrm{H}_{2} \mathrm{O}\right)_{2}\right]^{2+}$.

\begin{tabular}{|c|c|c|c|c|}
\hline \multirow[b]{2}{*}{ Transition } & \multicolumn{2}{|c|}{$\mathrm{Ni}(\mathrm{Py})_{4} \mathrm{Cl}_{2}$} & \multicolumn{2}{|c|}{$\left[\mathrm{Ni}(\mathrm{Py})_{4}\left(\mathrm{H}_{2} \mathrm{O}\right)_{2}\right]^{2+}$} \\
\hline & $\bar{E}^{\text {cacla }}$ & $E^{\operatorname{expb}}$ & $E^{\text {calcc }}$ & $E^{\operatorname{expb}}$ \\
\hline${ }^{3} B_{1 g} \rightarrow{ }^{3} E_{g}$ & 11,570 & 8,993 & 9,442 & 9,750 \\
\hline$\rightarrow^{3} B_{2 g}$ & 12,176 & 11,682 & 13,896 & 11,780 \\
\hline$\rightarrow^{1} A_{1 g}$ & $12,447)$ & 12,642 & 10,620 & 13,160 \\
\hline$\rightarrow^{1} B_{1 g}$ & $12,458\}$ & 12,804 & $13,011\}$ & 13,390 \\
\hline$\rightarrow{ }^{3} A_{2 g}$ & 18,873 & 15,267 & 15,485 & 15,760 \\
\hline$\rightarrow{ }^{3} E_{g}$ & 18,216 & 16,807 & 18,955 & 17,390 \\
\hline$\rightarrow^{1} A_{1 g}$ & $21,458)$ & 21834 & 24,116 ) & ח2 10 \\
\hline$\rightarrow^{1} E_{g}$ & $23,764\}$ & 21,834 & $21,975\}$ & 22,100 \\
\hline$\rightarrow^{1} B_{2 g}$ & 24,302 & 23,923 & 26,499 & - \\
\hline$\rightarrow^{1} E_{g}$ & 27,421 & - & 29,882 & - \\
\hline$\rightarrow^{1} A_{2 g}$ & 28,857 & - & 24,862 & - \\
\hline$\rightarrow{ }^{3} A_{2 g}$ & 29,163 & - & 27,318 & - \\
\hline$\rightarrow{ }^{3} E_{g}$ & 29,401 & 27,174 & 28,470 & 28,000 \\
\hline
\end{tabular}

${ }^{a}$ Racah parameters: $B=828 \mathrm{~cm}^{-1} ; C=3088 \mathrm{~cm}^{-1}$ [28].

${ }^{b}$ Experimental data from [28].

${ }^{c}$ Racah parameters: $B=784 \mathrm{~cm}^{-1} ; C=3525 \mathrm{~cm}^{-1}$ [28].

high-spin ${ }^{6} A_{1 g}$ is the exact ground state [31]. However, in the case of $\mathrm{CoP}$, the ground state is found to be quartet [32], and for $\mathrm{FeP}$, the ground state has been shown to be a quintet [33]. In both cases, the restricted $\mathrm{Cl}$ when taken into account partially improves the situation in the respect that the energies of the states with the required ground-state spin become lower; however, the ground state remains the high-spin one even with the extensive CI [33]. The EHCF method when applied to metal porphyrins improves the situation significantly. For the planar geometries of the three considered metal porphyrins, in all the cases, we reproduce the experimental ground-state spin and symmetry. An obvious difference between our calculation and those performed by the ab initio methods is the variance in the effective charges of the central ions. The ab initio works [31-33] report on the metal charges of about $+1.5 e$ In our (ultimately a CNDO) calculation on the $l$-system, the positive charge on the metal atoms does not exceed $+1 e$. However, in view of the low contribution the Coulomb interaction between the ligands and the $d$-shell gives to the actual crystal field induced onto the latter, the difference in the charge distribution reported above cannot account for the successful calculation of the ground-state spin (see below).

\section{General Discussion and Conclusion}

In the present article, we describe the effective Hamiltonian/crystal field (EHCF) method and analyze the results of its application to the calculation of the $d$-level splitting and $d-d$ spectra in a series of transition-metal complexes (TMC). The EHCF method allows one to perform systematic calculations of the ligand field for various ligand environments. The results of these calculations are in a fair agreement with the experimental data, particularly in the respect of the spin multiplicity of the ground states of the complexes. We were even able to solve some long-term problems in the transitionmetal quantum chemistry by reproducing in a consistent manner the experimental ground states for iron(II) porphyrin and for cobalt(II) porphyrin, which either was not possible in the Hartree-Fock approximation or required very long $\mathrm{CI}$ expansions.

The base for this success was, of course, a careful avoiding of the snares the SCF approximation 
TABLE $V$

Contributions to the crystal field in the

$\mathrm{ML}_{4} \mathrm{Z}_{2}$ complexes.

\begin{tabular}{|c|c|c|c|c|}
\hline \multicolumn{2}{|c|}{ Charge transfer $(i \rightarrow f)$} & \multirow[b]{2}{*}{$\Delta E_{i f}(\mathrm{eV})$} & \multirow[b]{2}{*}{$\left|\beta_{i f}\right|(\mathrm{eV})$} & \multirow{2}{*}{$\begin{array}{l}\beta^{2} / \Delta E \\
\left(\mathrm{~cm}^{-1}\right)\end{array}$} \\
\hline$\vec{i}$ & $f$ & & & \\
\hline \multicolumn{5}{|l|}{$\mathrm{Fe}(\mathrm{py})_{4}(\mathrm{NCS})_{2}$} \\
\hline$\sigma_{\mathrm{NCS}}$ & $d_{z^{2}}$ & 13.0 & 2.54 & 3980 \\
\hline$\sigma_{\mathrm{NCS}}$ & & 11.1 & 1.35 & 1330 \\
\hline$\sigma_{\mathrm{NCS}}$ & & 9.6 & 2.12 & 1780 \\
\hline$\sigma_{\mathrm{py}}$ & $d_{x^{2}-y^{2}}$ & 15.6 & 1.76 & 1600 \\
\hline$\sigma_{\mathrm{py}}$ & & 10.0 & 1.70 & 2340 \\
\hline \multicolumn{5}{|l|}{$\mathrm{Ni}\left(\mathrm{NH}_{3}\right)_{4}(\mathrm{NCS})_{2}$} \\
\hline $2 a_{1 g}$ & $d_{z^{2}}$ & 29.50 & 1.19 & 385 \\
\hline $3 a_{1 g}$ & & 18.56 & 0.72 & 223 \\
\hline $4 a_{1 g}$ & & 13.99 & 0.65 & 247 \\
\hline $5 a_{1 g}$ & & 8.40 & 2.68 & 6916 \\
\hline $6 a_{1 g}$ & & 6.00 & 1.28 & 2211 \\
\hline $1 b_{1 g}$ & $d_{x^{2}-y^{2}}$ & 29.15 & 1.83 & 923 \\
\hline $2 b_{1 g}$ & & 8.62 & 2.94 & 8114 \\
\hline $1 b_{2 g}$ & $d_{x y}$ & 15.18 & 0.83 & 369 \\
\hline $1 e_{g}$ & $d_{x z}, d_{y z}$ & 14.10 & 0.58 & 190 \\
\hline $2 e_{g}$ & & 10.16 & 0.35 & 97 \\
\hline $4 e_{g}(\mathrm{HUMO})$ & & 1.75 & 0.20 & 192 \\
\hline \multicolumn{5}{|l|}{$\mathrm{Ni}(\mathrm{Py})_{4} \mathrm{Cl}_{2}$} \\
\hline $5 a_{1 g}(s \mathrm{Cl})$ & $d_{z^{2}}$ & 18.83 & 1.53 & 1009 \\
\hline $6 a_{1 g}\left(p_{\sigma} N, C l\right)$ & & 14.20 & 0.87 & 430 \\
\hline $9 a_{1 g}\left(p_{\sigma} \mathrm{Cl}, \mathrm{N}\right)$ & & 4.48 & 2.39 & 10292 \\
\hline $4 b_{1 g}(s N)$ & $d_{x^{2}-y^{2}}$ & 22.26 & 1.45 & 759 \\
\hline $6 b_{1 g}\left(p_{\sigma} N\right)$ & & 12.61 & 1.98 & 2519 \\
\hline $7 b_{1 g}\left(s \mathrm{~N}, p_{\sigma} \mathrm{N}\right)$ & & 6.60 & 2.58 & 8140 \\
\hline $2 b_{2 g}(\pi \mathrm{Py})$ & $d_{x y}$ & 9.39 & 0.74 & 466 \\
\hline $7 e_{g}\left(p_{\pi} \mathrm{Cl}\right)$ & $d_{x z}, d_{y z}$ & 3.28 & 0.47 & 535 \\
\hline \multicolumn{5}{|l|}{$\left[\mathrm{Ni}(\mathrm{Py})_{4}\left(\mathrm{H}_{2} \mathrm{O}\right)_{2}\right]^{2+}$} \\
\hline $4 a_{g}(s \mathrm{~N}, s \mathrm{O})$ & $d_{z^{2}}$ & 30.43 & 1.70 & 766 \\
\hline $10 a_{g}\left(s \mathrm{Ni}, p_{\sigma} \mathrm{N}\right)$ & & 12.69 & 1.04 & 694 \\
\hline $13 a_{g}\left(p_{\sigma} \mathrm{O}\right)$ & & 10.27 & 2.16 & 3670 \\
\hline $9 a_{g}\left(p_{\sigma} N\right)$ & $d_{x^{2}-y^{2}}$ & 20.20 & 1.46 & 848 \\
\hline $14 a_{g}\left(p_{\sigma} \mathrm{O}, p_{\sigma} \mathrm{N}\right)$ & & 10.18 & 2.06 & 3356 \\
\hline $16 a_{g}\left(p_{\sigma} N\right)$ & & 5.32 & 2.32 & 8170 \\
\hline $3 b_{1 g}\left(p_{\pi} \mathrm{N}\right)$ & $d_{x y}$ & 7.90 & 0.69 & 489 \\
\hline
\end{tabular}

contains. It is well known that the SCF approximation overestimates the contribution of the chargetransfer states as compared to the exact (correlated) ground state [34]. We accepted as a fact that this shortcoming at least for the equilibrium geometries of the normal organic molecules can be
TABLE VI

$$
d-d \text { excitation energies }\left(\mathrm{cm}^{-1}\right) \text { of FeP. }
$$

\begin{tabular}{|c|c|c|c|c|}
\hline \multicolumn{5}{|c|}{ FeP, ground state ${ }^{3} E_{g}$ (for the symmetry $D_{4 h}$ ) } \\
\hline Tripl & & Sing & lets & Quintets \\
\hline $\begin{aligned}{ }^{3} E_{g} & \rightarrow{ }^{3} A_{2 g} \\
& \rightarrow{ }^{3} B_{2 g} \\
& \rightarrow{ }^{3} E_{g} \\
& \rightarrow{ }^{3} A_{2 g} \\
& \rightarrow{ }^{3} E_{g} \\
& \rightarrow{ }^{3} E_{g} \\
& \rightarrow{ }^{3} A_{1 g} \\
& \rightarrow{ }^{3} B_{1 g} \\
& \rightarrow{ }^{3} B_{2 g} \\
& \rightarrow{ }^{3} A_{2 g} \\
& \rightarrow{ }^{3} E_{g} \\
& \rightarrow{ }^{3} B_{1 g} \\
& \rightarrow{ }^{3} A_{1 g} \\
& \rightarrow{ }^{3} E_{g} \\
& \rightarrow{ }^{3} E_{g} \\
& \rightarrow{ }^{3} B_{2 g} \\
& \rightarrow{ }^{3} A_{1 g} \\
& \rightarrow{ }^{3} B_{2 g} \\
& \rightarrow{ }^{3} A_{2 g} \\
& \rightarrow{ }^{3} B_{1 g} \\
& \rightarrow{ }^{3} E_{g} \\
& \rightarrow{ }^{3} A_{2 g}\end{aligned}$ & $\begin{array}{r}1,121 \\
2,297 \\
8,160 \\
18,954 \\
19,317 \\
19,766 \\
19,870 \\
20,270 \\
20,590 \\
21,310 \\
21,892 \\
22,442 \\
22,578 \\
23,567 \\
23,814 \\
23,941 \\
25,197 \\
26,193 \\
33,285 \\
35,379 \\
35,934 \\
41,085\end{array}$ & $\begin{array}{l}\rightarrow^{1} A_{1 g} \\
\rightarrow^{1} E_{g} \\
\rightarrow^{1} B_{1 g} \\
\rightarrow^{1} B_{2 g} \\
\rightarrow^{1} B_{2 g} \\
\rightarrow^{1} A_{1 g} \\
\rightarrow^{1} E_{g} \\
\rightarrow^{1} A_{2 g} \\
\rightarrow^{1} A_{1 g} \\
\rightarrow^{1} E_{g} \\
\rightarrow^{1} B_{1 g} \\
\rightarrow^{1} B_{2 g} \\
\rightarrow^{1} A_{2 g} \\
\rightarrow^{1} E_{g} \\
\rightarrow^{1} B_{1 g} \\
\rightarrow^{1} B_{2 g} \\
\rightarrow^{1} E_{g} \\
\rightarrow^{1} A_{1 g} \\
\rightarrow^{1} E_{g} \\
\rightarrow^{1} A_{1 g} \\
\rightarrow^{1} A_{2 g} \\
\rightarrow^{1} B_{1 g}\end{array}$ & $\begin{array}{r}4,898 \\
6,570 \\
8,318 \\
8,704 \\
12,308 \\
13,467 \\
14,803 \\
24,203 \\
24,765 \\
25,090 \\
26,470 \\
27,193 \\
29,242 \\
30,294 \\
31,245 \\
31,621 \\
31,627 \\
31,687 \\
32,110 \\
38,805 \\
41,346 \\
43,435\end{array}$ & $\begin{array}{lr}\rightarrow{ }^{5} E_{g} & 6,137 \\
\rightarrow{ }^{5} A_{1 g} & 6,840 \\
\rightarrow{ }^{5} B_{2 g} & 7,806 \\
\rightarrow{ }^{5} B_{1 g} & 28,650\end{array}$ \\
\hline
\end{tabular}

covered by some parametrization (e.g., by CNDO). The reason is that in a normal organic molecule one can never distinguish the multiplet terms of the separate component atoms. This is the state of things which one could call "organic quantum chemistry." By contrast, the $d$-shell of a transitionmetal ion in a complex largely retains the system of the terms that it had in the free state. When the $d$-shell is included in the general SCF-MO-LCAO procedure, the singly, doubly, etc., positively and negatively ionized states of this shell are produced and overestimated by this scheme. One can try to correct these errors by performing $\mathrm{CI}$ which suppresses the configurations corresponding to the excessively charged atoms. However, these $\mathrm{Cl}$ expansions are performed in the basis of the MO-LCAO Slater determinants and it takes too many of them to suppress the configurations where the atoms are excessively charged. Indeed, in the case of the $\mathrm{H}_{2}$ molecule, in order to suppress the charge fluctua- 
TABLE VII

$d-d$ excitation energies $\left(\mathrm{cm}^{-1}\right)$ for the CoP.

\begin{tabular}{|c|c|c|}
\hline \multicolumn{3}{|c|}{ CoP, ground state ${ }^{2} A_{1 g}$ (for symmetry $D_{4 n}$ ) } \\
\hline & Doublets & Quartets \\
\hline 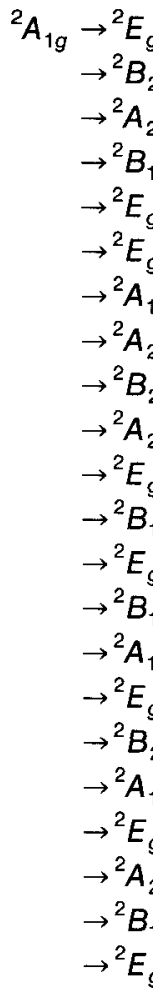 & $\begin{array}{r}2,845 \\
9,494 \\
15,790 \\
16,848 \\
17,462 \\
17,850 \\
18,594 \\
18,711 \\
20,738 \\
21,562 \\
22,032 \\
22,492 \\
24,998 \\
32,732 \\
34,814 \\
35,339 \\
35,707 \\
36,084 \\
36,085 \\
36,970 \\
40,625 \\
40,979\end{array}$ & $\begin{array}{lr}\rightarrow{ }^{4} E_{g} & 5,158 \\
\rightarrow{ }^{4} A_{2 g} & 5,404 \\
\rightarrow{ }^{4} B_{2 g} & 6,008 \\
\rightarrow{ }^{4} E_{g} & 11,524 \\
\rightarrow^{4} B_{1 g} & 23,989 \\
\rightarrow^{4} E_{g} & 26,328 \\
\rightarrow^{4} A_{2 g} & 32,891\end{array}$ \\
\hline
\end{tabular}

TABLE VIII

Contributions to the crystal field in porphyrins.

\begin{tabular}{lcrrr}
\hline Complex & $\begin{array}{c}\text { Charge transfer } \\
(i \rightarrow f)\end{array}$ & $\begin{array}{r}\Delta E_{i f} \\
(\mathrm{eV})\end{array}$ & $\begin{array}{r}\left|\beta_{i f}\right| \\
(\mathrm{eV})\end{array}$ & $\begin{array}{r}\beta^{2} / \Delta E \\
\left(\mathrm{~cm}^{-1}\right)\end{array}$ \\
\hline FeP & $p_{\sigma}(\mathrm{N}) \rightarrow d\left(a_{1 g}\right)$ & 11.8 & 1.26 & 1,082 \\
& $d\left(a_{1 g}\right) \rightarrow s_{\sigma}(\mathrm{N})$ & 9.9 & 1.43 & $-1,656$ \\
& $p_{\sigma}(\mathrm{N}) \rightarrow d\left(b_{1 g}\right)$ & 21.0 & 3.16 & 3,845 \\
& $p_{\sigma}(\mathrm{N}) \rightarrow d\left(b_{1 g}\right)$ & 12.5 & 2.03 & 2,672 \\
& $p_{\sigma}(\mathrm{N}) \rightarrow d\left(b_{1 g}\right)$ & 8.3 & 3.68 & 13,219 \\
$\mathrm{CoP}$ & $s_{\sigma}(\mathrm{N}) \rightarrow d\left(b_{1 g}\right)$ & 19.2 & 2.59 & 2,815 \\
& $p_{\sigma}(\mathrm{N}) \rightarrow d\left(b_{1 g}\right)$ & 10.7 & 1.68 & 2,127 \\
& $p_{\sigma}(\mathrm{N}) \rightarrow d\left(b_{1 g}\right)$ & 6.4 & 3.12 & 12,365 \\
\hline
\end{tabular}

tions (we follow here the terminology due to Malrieu [35]), the doubly excited configuration $\left(\sigma^{*}\right)^{2}$ must be added to the Hartree-Fock ground state $\sigma^{2}$. In the context of the TMC that means that at least two SCF-MOS (bonding and antibonding ones) must be included into the active space for each $d$-AO in order to suppress the charge fluctuations in the $d$-shell. In effect, each of the $d$-AOs contributes to a much larger number of the occupied and empty MOs and, strictly speaking, all of them must be included in order to suppress the fluctuations.

In the EHCF method, we, from the very beginning, do not allow the overcharged configurations of the $d$-shell to appear. The only allowed chargetransfer configurations are those where one electron is transferred from the $d$-shell or into it. In that respect, we proceed in a line with [13]. We, however, do one more step and project out the configurations with the charge transfer. That gives us the effective crystal-field splitting of the oneelectron $d$-levels and allows us to retain the multiplets of the free ions in our consideration. This correct form of the wave function for TMC does not have the charge fluctuations, and for that reason, it is enough to take into consideration a small active space-the configurations of the $d$-shell only.

Now let us turn to the limitations of the method and of its current implementation which also have to be mentioned. In the simple version of the EHCF method, we present here the electronic structure of the ligands treated within the CNDO approximation, which is, probably, enough in our case when we only need to reproduce the charge distribution in the ligands and to estimate their orbital energies at the fixed experimental geometries. It will not suffice for the geometry optimization when, at least, the corrected core-core repulsion is necessary. Another problem difficult to address with use of the current implementation of the EHCF method is the spin-polarization of the diamagnetic ligands by the paramagnetic central ions which would require exchange interaction to be included in the theory. This work is in progress now.

Another limitation can be, probably, considered as an advantage of the method. The resonance interaction between the $d$-shell and the ligands is taken into account perturbatively. It imposes an additional restriction on the energy denominators $\Delta E_{d i}$ and $\Delta E_{i d}$, which have to be positive and not too small. When it is not satisfied, we consider that as an alarm signal, indicating that the assumed electron distribution between the $d$-shell and the ligands is not correct and either some other electron distribution must be assumed or some charge-transfer states (or, in other words, the resolvent poles) intervene between the $d-d$ excitations. 
The latter is frequently the case for the low-valent metal centers like those in metal carbonyls [like $\left.\mathrm{Ni}(\mathrm{CO})_{4}\right]$. For them, the atomic configuration $s^{1} d^{n-1}$ has a low energy and is strongly admixed. Incidentally, the molecules of that type are never considered as derivatives of the free ions with, say, the configuration $d^{8}$ (in the $\mathrm{Ni}$ case) and they are not fit to the conventional CFT. Respectively, their electronic structure cannot be described by the EHCF method presented in this article.

\section{ACKNOWLEDGMENTS}

This method has been largely supported by the Russian Foundation for Fundamental Research (RFFR) through Grant 94-03-9902. It has been completed during respective visits of $\mathrm{A}$. L. T. to the Max-Planck-Institut für Physik komplexer Systeme, Dresden, and of A. V. S. to the Universität Hannover. The hospitality of these institutions is gratefully acknowledged as is the research fellowship granted to A. V. S. by Alexander von Humboldt Foundation. The generous support from the Organizing Committee of the International Workshop on Electronic Structure Methods for Truly Large Systems which made possible the visit of I. A. M. to Braunlage (Harz, Germany) and his participation in the work of the said workshop is gratefully acknowledged as well. The authors are grateful to the referee for the instructive comments.

\section{Appendix}

Here, we present a proof that the Slater determinant breaking the Aufbauprinzip cannot be a minimum of the Hartree-Fock energy functional. We must pay credit for this proof to Prof. V. I. Pupyshev of the Chemistry Department of the Moscow State (Lomonosoff) University as it had been given in the lectures that he used to deliver and seems to be never published [36].

Let us consider the one-electron reduced density matrix $\mathbf{R}$ for the one-determinant wave function:

$$
\Psi=\operatorname{det}\left[\phi_{1}, \phi_{2}, \ldots, \phi_{n}\right]
$$

where $\phi_{i}$ are occupied molecular spin-orbitals (MSO). $\mathbf{R}$ is the matrix of the projector on the $n$-dimensional subspace of the occupied MSo. This matrix can be presented in the following form:

$$
\mathbf{R}=[\phi][\phi]^{+},
$$

where $[\phi]$ is a column vector. It can be written also as

$$
\mathbf{R}=\sum_{i}|i\rangle\langle i|, \quad| i\rangle=\phi_{i} .
$$

The matrix $\mathbf{R}$ is idempotent and its trace is equal to total number of electrons:

$$
\mathbf{R}^{+}=\mathbf{R}, \quad \mathbf{R}^{2}=\mathbf{R}, \quad \operatorname{Sp} \mathbf{R}=N .
$$

In the Hartree-Fock approximation, the energy function has the form

$$
E(\mathbf{R})=\mathrm{Sp}[\mathbf{h R}]+\mathrm{Sp}[\mathbf{R J}(\mathbf{R})-\mathbf{R K}(\mathbf{R})] / 2 .
$$

Here, $\mathbf{h}$ is the matrix of one-electron part of the total Hamiltonian; the matrix elements of the Coulomb $(\mathrm{J})$ and exchange $(\mathrm{K})$ operators in the basis of orthonormalized MSO $\chi_{k}$ have the form

$$
\begin{aligned}
{[\mathbf{J}(\mathbf{R})]_{i j} } & =\sum_{k, l}(i j \mid k l) R_{l k} \\
{[\mathbf{K}(\mathbf{R})]_{i j} } & =\sum_{k, l}(i l \mid k j) R_{l k} .
\end{aligned}
$$

With

$$
\mathbf{G}(\mathbf{R}) \equiv \mathbf{J}(\mathbf{R})-\mathbf{K}(\mathbf{R})
$$

one has

$$
E(\mathbf{R})=\mathrm{Sp}[\mathbf{h R}]+\mathrm{Sp}[\mathbf{R G}(\mathbf{R})] / 2 .
$$

The Hartree-Fock equation for the density matrix has the following form:

$$
\mathbf{F}(\mathbf{R}) \mathbf{R}=\mathbf{R F}(\mathbf{R}),
$$

where $\mathbf{F}(\mathbf{R})=\mathbf{h}+\mathbf{G}(\mathbf{R})$ is the matrix of the Fock operator. In the basis of canonical orbitals, this matrix is diagonal and its eigenvalues are the energies of the MSO:

$$
\mathbf{F}|i\rangle=\varepsilon_{i}|i\rangle .
$$

Now, we can formulate the following theorem. If the solution of the Hartree-Fock equations corresponds to the absolute minimum of the energy functional, then the Aufbauprinzip is valid, i.e., the energy of any unoccupied MSO is larger than the energy of any occupied one. 
Proof. Let us assume that the density matrix $\mathbf{R}$ corresponds to the absolute minimum of the energy functional. From the variational principle, it follows that for all $\mathbf{R}^{\prime}$ the inequality $E\left(\mathbf{R}^{\prime}\right) \geq E(\mathbf{R})$ holds. Set

$$
\mathbf{R}^{\prime}=\mathbf{R}+\Delta,
$$

where

$$
\Delta=|p\rangle\langle p|-| q\rangle\langle q|,
$$

and $p$ is an unoccupied MSO, and $q$ is an occupied MSO. Then,

$$
\begin{aligned}
E\left(\mathbf{R}^{\prime}\right)= & E(\mathbf{R}+\Delta)=\operatorname{Sp}[\mathbf{h}(\mathbf{R}+\Delta)] \\
& +\operatorname{Sp}[(\mathbf{R}+\Delta) \mathbf{G}(\mathbf{R}+\Delta)] / 2 \\
= & E(\mathbf{R})+\operatorname{Sp}[h \Delta]+\operatorname{Sp}[\Delta \mathbf{G}(\mathbf{R})] \\
& +\operatorname{Sp}[\Delta \mathbf{G}(\Delta)] / 2 .
\end{aligned}
$$

Here, we have used the relation

$$
\mathrm{Sp}[\mathbf{A G}(\mathbf{B})]=\operatorname{Sp}[\mathbf{B G}(\mathbf{A})] .
$$

Commuting the matrices under the Spur in the third term of Eq. (A11) and taking into account the definition of $\mathbf{F}(\mathbf{R})$, we have

$$
\begin{aligned}
E\left(\mathbf{R}^{\prime}\right)=E(\mathbf{R})+\operatorname{Sp}[\mathbf{F}(\mathbf{R}) \Delta] & \\
& +\operatorname{Sp}[\Delta \mathbf{G}(\Delta)] / 2 .
\end{aligned}
$$

Since $|p\rangle$ and $|q\rangle$ are the canonical orbitals, the following holds:

$$
\mathrm{Sp}[\mathbf{F}(\mathbf{R}) \Delta]=\varepsilon_{p}-\varepsilon_{q},
$$

where $\varepsilon_{p}$ and $\varepsilon_{q}$ are the corresponding orbital energies. Thus, we have

$$
\begin{aligned}
E\left(\mathbf{R}^{\prime}\right)=E(\mathbf{R})+ & \left(\varepsilon_{p}-\varepsilon_{q}\right) \\
& -\operatorname{Sp}[|q\rangle\langle q| \mathbf{G}(|p\rangle\langle p|)] .
\end{aligned}
$$

Let us evaluate the sign of the last term in this formula:

$$
\mathrm{Sp}[|q\rangle\langle q| \mathbf{G}(|p\rangle\langle p|)]=(q q \mid p p)-(q p \mid p q)>0 .
$$

Finally, we have

$$
\begin{aligned}
E\left(\mathbf{R}^{\prime}\right) & =E(\mathbf{R})+\left(\varepsilon_{p}-\varepsilon_{q}\right)-a^{2} \\
\varepsilon_{p}-\varepsilon_{q} & =E\left(\mathbf{R}^{\prime}\right)-E(\mathbf{R})+a^{2}>0,
\end{aligned}
$$

since $E\left(\mathbf{R}^{\prime}\right)-E(\mathbf{R}) \geq 0$ because of the variational principle.

\section{References}

1. M. C. Zerner and A. D. Bacon, Theor. Chim. Acta. 53, 21 (1979).

2. M. C. Zerner, G. H. Loew, R. F. Kirchner, and U. T. Mueller-Westerhoff, J. Am. Chem. Soc. 102, 589 (1980).

3. W. D. Edwards, B. Weiner, and M. C. Zerner, J. Phys. Chem. 92, 6188 (1988).

4. W. D. Edwards, B. Weiner, and M. C. Zerner, J. Am. Chem. Soc. 108, 2196 (1986).

5. M. Kotzian, N. Rösch, H. Schröder, and M. C. Zerner, J. Am. Chem. Soc. 111, 7687 (1989).

6. J. E. Newton and M. B. Hall, Inorg. Chem. 23, 4627 (1984).

7. J. E. Newton and M. B. Hall, Inorg. Chem. 24, 2573 (1985).

8. K. Pierloot and L. G. Vanquickenborne, J. Chem. Phys. 93, 4154 (1990).

9. M. Rosi, C. W. Bauschlicher, S. C. Langhoff, and H. Partridge, J. Phys. Chem. 94, 8656 (1990).

10. K. Morokuma, Inorg. Chem. 29, 3110 (1990).

11. H. Johansen and N. K. Andersen, Mol. Phys. 58, 965 (1986).

12. S. Y. Shashkin and W. A. Goddard III, Phys. Rev. B 33, 153 (1986).

13. G. J. M. Janssen and W. C. Nieuwpoort, Int. J. Quantum Chem. Symp. 22, 679 (1988).

14. A. V. Soudackov, A. L. Tchougréeff, and I. A. Misurkin, Theor. Chim. Acta 83, 389 (1992).

15. M. C. Böhm, Theor. Chim. Acta 60, 233 (1981).

16. J. P. Blaizot and G. Ripka, Quantum Theory of Finite Systems (MIT Press, Cambridge, MA, 1986); A. V. Soudackov, PhD Thesis (Karpov Institute of Physical Chemistry, Moscow, 1991) (in Russian).

17. C. K. Jorgensen, Modern Aspects of Ligand Field Theory (North-Holland, Amsterdam, 1971).

18. A. B. P. Lever, Inorganic Electronic Spectroscopy (Elsevier, Amsterdam, 1986).

19. A. V. Soudackov, A. L. Tchougréeff, and I. A. Misurkin, in Electron-Electron Correlation Effects in Low-Dimensional Conductors and Superconductors, A. A. Ovchinnikov and I. I. Ukrainskii, Eds. (Springer-Verlag, Berlin, 1991).

20. A. V. Soudackov, A. L. Tchougréeff $f_{r}$ and I. A. Misurkin, Zh. Fiz. Khim. 68, 1257 (1994) (in Russian); Russ. J. Phys. Chem. 68, 1135 (1994) (in English).

21. A. V. Soudackov, A. L. Tchougréeff, and I. A. Misurkin, Zh. Fiz. Khim. 68, 1265 (1994) (in Russian); Russ. J. Phys. Chem. 68, 1142 (1994) (in English).

22. A. L. Tchougréeff, A. V. Soudackov, I. A. Misurkin, H. Bolvin, and O. Kahn, Chem. Phys. 193, 19 (1995).

23. R. McWeeny, Methods of Molecular Quantum Mechanics (Academic Press, London, 1992).

24. J. A. Pople and D. L. Beveridge, Approximate Molecular Orbital Theory (McGraw-Hill, New York, 1970). 
25. D. W. Clack, N. S. Hush, and S. R. Yandle, J. Chem. Phys. 57, 3503 (1972).

26. M. Gerloch and R. G. Wooley, Progr. Inorg. Chem. 31, 371 (1984).

27. C. R. Hare and C. J. Ballhausen, J. Chem. Phys. 40, 792 (1964).

28. J. S. Merriam and J. R. Perrumareddi, J. Phys. Chem. 79, 142 (1975).

29. R. J. Doedens and L. F. Dahl, J. Am. Chem. Soc. 88, 4847 (1966).

30. A. Zalkin, D. H. Templeton, and T. Ueki, Inorg. Chem. 12, 1641 (1973)
31. A. Dedieu, M.-M. Rohmer, and A. Veillard, Adv. Quantum Chem. 16, 43 (1982).

32. H. Kashiwagi, T. Takada, S. Obara, E. Miyoshi, and K. Ohno, Int. J. Quantum Chem. 14, 13 (1978).

33. D. C. Rawlings, M. Gouterman, E. R. Davidson, and D. Feller, Int. J. Quantum Chem. 28, 773 (1985).

34. P. Fulde, Electron Correlations in Molecules and Solids (Springer-Verlag, 1993).

35. J.-P. Malrieu, in The Concept of the Chemical Bond, Part 2, Z. B. Maksic, Ed. (Springer-Verlag, Berlin, 1990).

36. V. I. Pupyshev, Additional Chapters of Molecular Quantum Mechanics. Course of Lectures (Unpublished). 